\title{
Synergy and Integration of Multiple Media to Enhance Exposure and Impact of Out-of-Home Advertising
}

\author{
Thérèse Roux and De la Rey van der Waldt
}

\begin{abstract}
For media planners to combine multiple media across platforms to maximize synergy is a critical challenge within integrated marketing communication. For this study, in-depth interviews were conducted with experienced media specialists in leading agencies to explore how they integrate different out-of-home advertising media platforms in IMC strategies. The feedback of these experts was then used to propose a model of strategies to enhance synergy within out-of-home advertising campaigns. This model is a first of its kind for planning out-of-home advertising media across platforms and bridges a gap in the existing literature on media synergy and out-of-home advertising media strategy.
\end{abstract}

\footnotetext{
T. Roux $(\bowtie)$

Tshwane University of Technology, Pretoria, South Africa

e-mail: rouxat@gmail.com

D.1.R. van der Waldt

North-West University, Mahikeng, South Africa

e-mail: Delarey.VanDerWaldt@nwu.ac.za 